\title{
14
}

\section{Provision of Broadband Video Conference via IN and B-ISDN Integration: Architectural and Modelling Issues}

\author{
F. Cuomo, M Listanti, F. Pozzi \\ INFOCOM Dept., University of Rome "La Sapienza" \\ Via Eudossiana, 18 - 00184 Rome, Italy; \\ Tel.: +39-6-44585472, Fax.:+39-6-4873300, \\ e-mail: franci@antares.ing.uniromal.it
}

\begin{abstract}
This paper deals with the integration between the Intelligent Network (IN) and the B-ISDN for the support of an advanced multimedia service, i.e. the Broadband Video Conference (B-VC). The focus of the paper is the proposal of a new role of the IN that, in a closed interaction with the B-ISDN, furnishes control functionality to handle complex service configuration.

In order to assign such a role, we distinguish different levels of control into the IN architecture and we propose functional models able to represent, at each level, a specific service view, suitable to th IN, and cooperating for a global service provisioning.

Some different options for an IN/B-ISDN interaction are discussed; in particular, the architectural aspects of a solution distributing the IN control logic between the Service Control Function (SCF) and the Service Switching Function (SSF) are described in details.

Finally we consider an actual multimedia service, the Broadband Video Conference, supported with the proposed approach, and we analyse the relevant performance behaviour.
\end{abstract}

Keywords

IN and B-ISDN, multimedia services, IN architecture. 


\section{INTRODUCTION}

The growing user needs and the emerging advanced computer applications require, in addition to networks with high bandwidth capabilities, a powerful signalling system able to manage and to coordinate sophisticated network configuration scenarios.

Up to now the standardisation bodies have defined two B-ISDN signalling protocol releases: the Signalling Capability Set 1 (SCS 1) and Signalling Capability Set 2 (SCS 2) [ITU-T Q.2931, 1994] [ITU-T Q.2971, 1994].

The SCS 1 supports simple switched services consisting of single-connection, bi-directional, point-to-point calls. The objective of SCS 2 was the handling of: multiparty calls, several connections in a single call, correlation among different media, heterogeneous terminals and dynamic change of call topology and connections parameters. This wide objective suggested to split the signalling protocol definition activity in steps. The first step gave rise the SCS 2.1 , that is, at present, the unique stable signalling capability set. The main innovation within SCS 2.1 is the point-to-multipoint connection handling.

Unfortunately, there still exists a remarkable gap between the standardised B-ISDN control capabilities and the requirements of new telecommunication services based on multipoint-to-multipoint call configurations, as in the case of the Broadband Video Conference (B-VC). A solution to rapidly fill up this gap is the integration of the B-ISDN and the IN paradigms. This approach has been proposed in previous papers [Maastricht, 1995] [Wakamoto, 1995] [Mukasa, 1995] [Carmagnola, 1996] and is the focus of an European ACTS project, named INSIGNIA [AC068 INSIGNIA deliverables].

As well known, IN allows a flexible introduction of new capabilities, and facilitates and accelerates, in a cost effective manner, service implementation and provisioning in a multi-vendor environment. The flexibility of this paradigm mainly lies in the separation between the control functionality relevant to the transport plane and the ones relevant to the service provisioning.

The classical IN service provisioning is based on a direct interaction between the IN logic, implementing supplementary aspects of the service, and the bearer service furnished at the transport level [ITU Q.1211-Q.1218, 1995]. In the IN/B-ISDN integrated approach, the IN provides a platform to enrich the functionalities furnished by the B-ISDN, in order to realise the handling of several basic calls/connections in a coordinate manner, according to the service logic requirements [Cuomo, 1996].

Figure 1 represents a logical scheme showing how this new functionality, here called Bearer Connections Coordinator (BCC), is inserted within the integrated IN/B-ISDN functional architecture.

By considering the present B-ISDN signalling capabilities, the BCC must be entirely located in the IN domain. In the future, the growing of the B-ISDN signalling capabilities could bring a reduction of the functionalities realised in the 
IN domain and a migration of some functions within the B-ISDN one. It is also to be noted that we refer to B-ISDN call and connection as a unique entity since, for the time being, they are handled at the B-ISDN level in a monolithic way.

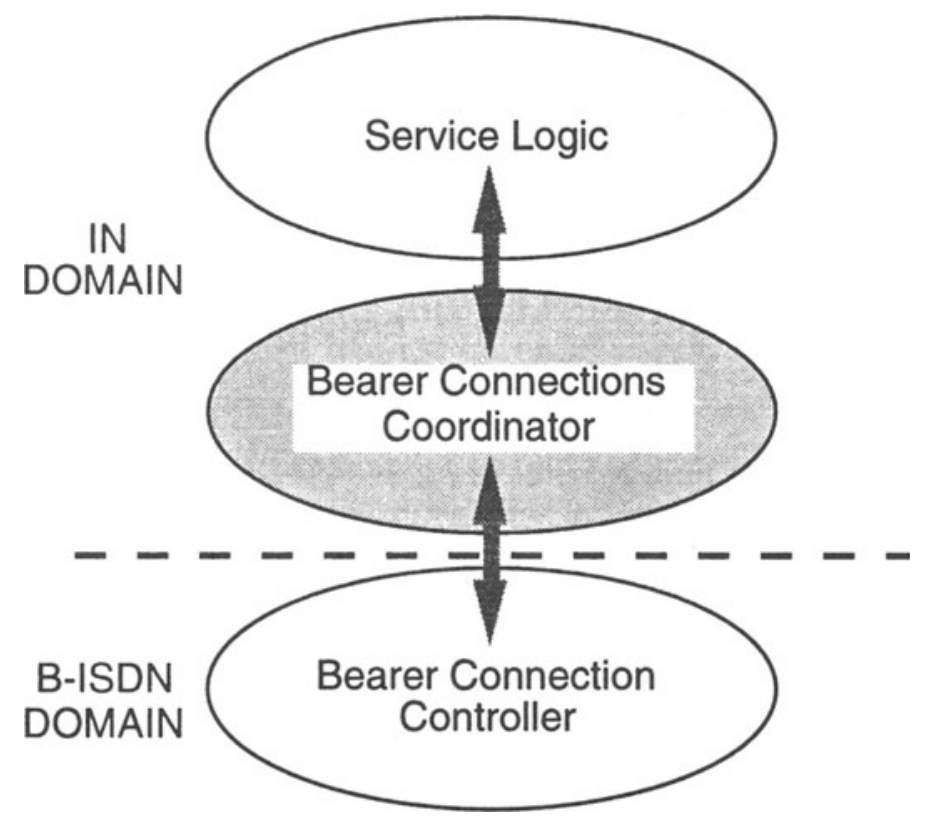

Figure 1 Functional organisation of the integrated IN/B-ISDN paradigm.

In this paper we discuss the functionality of the IN Bearer Connections Coordinator with particular reference to the provisioning of the Broadband Video Conference service. The solution here presented, derived from one of the main outcomes of the INSIGNIA project, foresees that the BCC functionality is shared between the Service Control Function (SCF) entity, located in the Service Control Point (SCP) and the Service Switching Function (SSF) entity, located in the Service Switching Point (SSP).

The coordination between the two entities is carried out by means of two service representation models: the former, named Global Logic Service Configuration (GLSC) is resident in the SCF; the latter, named session, is resident in the SSF.

This solution establishes a separation between the role of the SSF, that, according to the classical IN paradigm, furnishes functionalities independent of the specific service, and the role of the SCF that realises the service dependent functionalities. Moreover, as it will be described in the following, this solution allows a very efficient SCF/SSF interaction based on a high level service view. The SSF translates such a view into multiple views of lower level (i.e. the Basic 
Call State Models - BCSMs), for the interaction with the B-ISDN control functionalities.

As a matter of example, in the second part of the paper, the proposed approach is applied for the provision of the Broadband Video Conference service, which requires very advanced control capabilities to handle the relevant network configuration.

The organisation of the paper is the following: Sec. 2 describes the functional levels in which the BCC is splitted and the relevant service representation models (i.e. the GLSC and the session). In Sec. 3 the IN approach is applied for the provision of the B-VC service, showing two architectural solutions. A brief performance evaluation is also carried out.

\section{THE BEARER CONNECTIONS COORDINATOR IN THE IN DOMAIN}

The fundamental requirement, in the design of an integrated IN/B-ISDN system architecture, is that different basic B-ISDN calls/connections have to be coordinated by the IN, in order to provide the required user plane configuration.

To this aim, in the framework of the INSIGNIA project, a functional architecture structured in four control domains has been developed [AC068 INSIGNIA Deliverable]: i) the service control domain, comprising the overall control of the IN service; ii) the session control domain, where the association of different B-ISDN calls for the realisation of a single IN service is handled; iii) the call control domain where each single B-ISDN call is controlled; iv) the connection control domain, where the physical switching resources involved in a B-ISDN call are controlled.

Figure 2 shows the relationships among these four domains and their mapping on the IN functional entities. Moreover, in the same figure, the two functional domains where the $\mathrm{BCC}$ functionality is implemented are singled out.

Within the service control domain, the BCC is responsible for the global service control and of the relevant network configuration; this goal is achieved by means of the interaction between the service logic inside the SCF, responsible of the service execution, and a high level representation of all the network components and of their relationships (GLSC).

As far as the session control domain is concerned, the BCC is dedicated to the control and the coordination of all the calls/connections supporting a specific service. By considering a single service instance, two main architectural solutions can be applied: i) a centralised approach, the session control function is located in a single SSF; ii) a distributed approach, the session control function is distributed among many SSFs.

\subsection{The session control domain}

The session control domain represents the lower level of coordination of basic B-ISDN calls/connections involved in the provisioning of a single service. As shown in Figure 2, the B-ISDN call/connections are modelled independently by 
means of the classical BCSM inside the Call Control Function (CCF). The connection coordination is obtained by developing, within the IN Switching Manager (IN-SM) entity inside the SSF, an enhanced Switching State Model (SSM) to be offered to the SCF control [AC068 INSIGNIA Deliverable].

This enhanced model, indicated as session, is realised by means of a suitable object modelling, and it is the tool needed to represent the connections, parties and relationships involved in the service in a specific SSF.

The IN Switching Manager handles the call events reported by the BCSMs and correlates them in the context of a session by sending appropriate messages to the SCF. In the other direction, the IN SM receives commands from the SCF and sends appropriate messages to the BCSMs (Figure 2).

In the centralised approach, a single session comprises all the B-ISDN calls/connections involved in the service; this means that a single SSP controls the whole set of B-ISDN connections. This solution, especially in multiparty services, where the number of involved connections could be significant, gives rise to an inefficient resource utilisation, both from a transport and a control point of view.

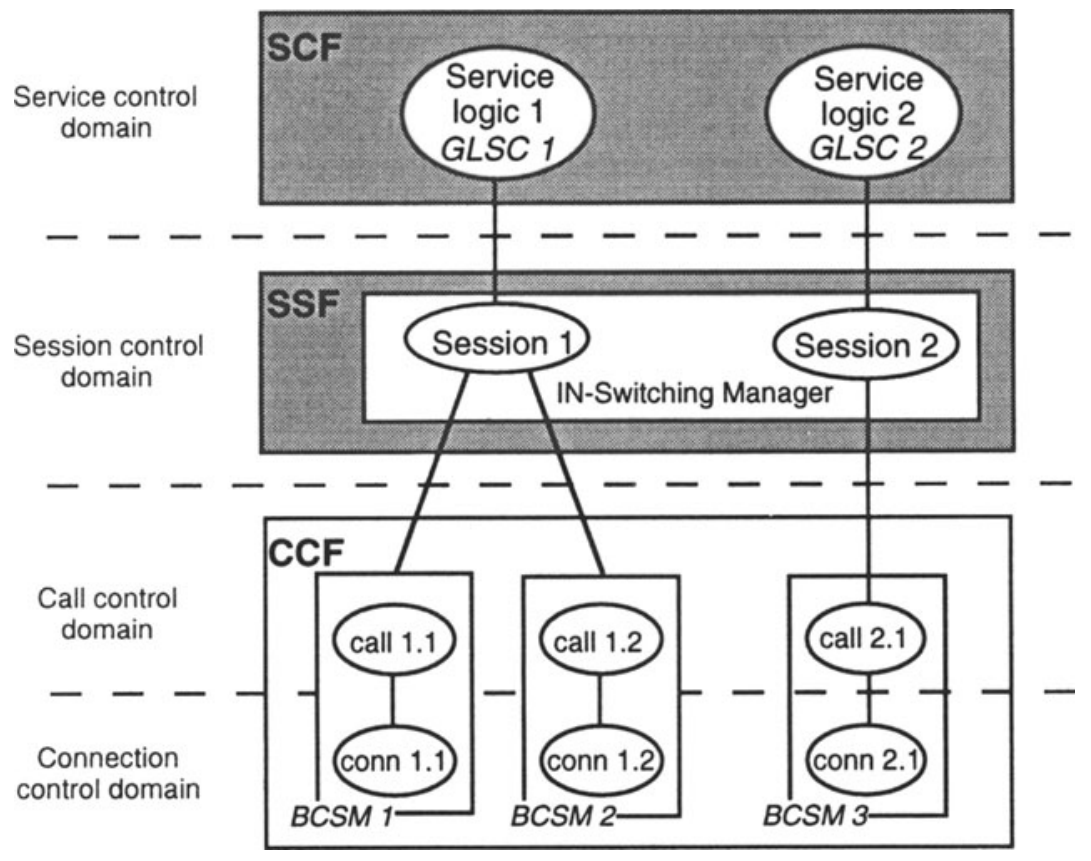

Figure 2 Control domains definition within IN/B-ISDN functional architecture.

A more suitable solution, the distributed one, foresees that, if needed, there could be more than one SSF involved in the same service instance. This means that the calls/connections composing the service configuration are controlled by different 
SSPs. The controlling SSPs are selected with the aim of minimising the usage of the network resources.

The distributed solution implies that the session contained in each SSF represents only a partial view of the service configuration. The merging of these partial views is performed at the service control domain by the SCF.

To fully exploit the potentialities of the session concept, two new control capabilities are introduced within the session control domain:

1. SCP-initiated call; the SCP can order the SSP to set-up a call between two users (i.e. a point-to-point connection). This IN functionality supplies the "Third Party Call Initiated" procedure; in this procedure separate paths of a connection towards two remote users are set-up and linked by a third party. This functionality can also be used to set-up a point-to-multipoint connection among a root user and a multiplicity of leaf users. In this case, the root is connected to the SSP by means of a single unidirectional point-to-point connection, while the point-to-multipoint part of the connection starts from the SSP.

2. SCP-session activation; this command is used to activate a session (in a generic SSP) on behalf the SCP. This functionality allows the control of the connections involved in a service through a multiplicity of sessions distributed in several SSPs. It is to be noted that this capability differs from that used in the classical IN approach, where the activation of a SSM instance is always due to a user request.

The proposed object model of a session is shown in Figure 3. It contains objects that are abstractions of switching and transmission resources [AC068 INSIGNIA Deliverable].

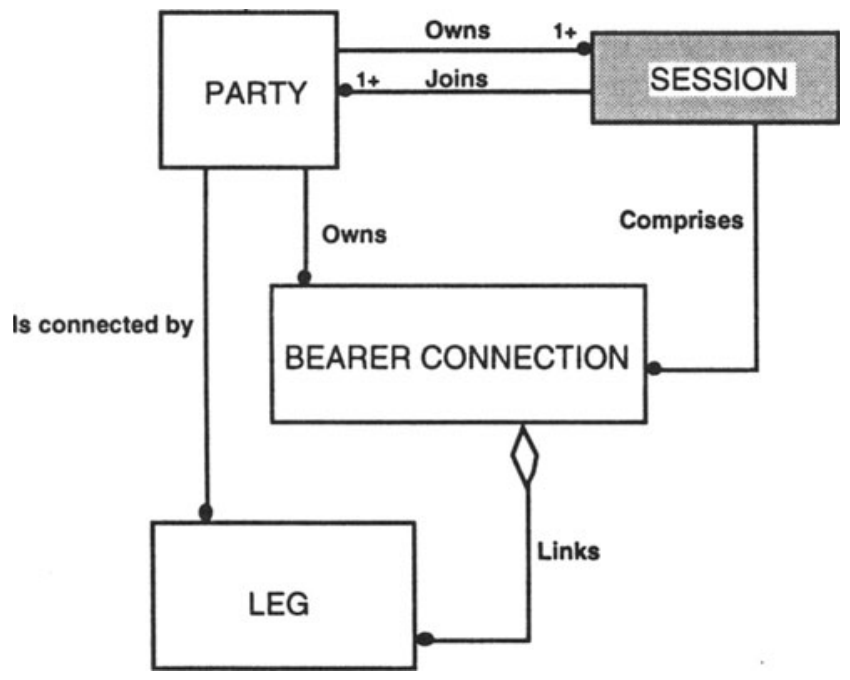

Figure 3 The session object model. 
The session object represents the session instance. Several parties, represented by the party object, can join a session. A party can be either an end user or a network component (e.g. the SCP, when it is necessary to represent SCP-initiated actions). One of the parties joining a session is the session owner. During the service evolution, new parties can be added to a session or joined parties can be removed from a session.

The bearer connection object represents the bearer connection processing and is potentially in relationship with several legs. The leg object models the processing of the communication path towards a party. The multiplicity of the aggregations between the legs and a bearer connection determines the connection topology: if a bearer connection contains exactly two legs, it is a point-to-point connection; if there are more than two legs per bearer connection, we have a point-to-multipoint connection.

Moreover, each object could also be denoted by one or more attributes regarding some of its specific characteristics. In particular, we can distinguish static attributes and dynamic ones. The static attributes denote fixed characteristics of the object assigned in the active service instance. The dynamic attributes change during the service evolution and are used by the IN logic to follow the dynamic modifications of the service configuration.

The proposed model is a powerful tool to handle the interaction between the service control domain and the B-ISDN control function. It shows each single service component, the state reached by each of them and their relationships. Moreover, this model allows the utilisation of a high level language between the SCF and the SSF (i.e. an enhanced INAP protocol called B-INAP [AC068 INSIGNIA Deliverable]). As a matter of example, the SCF can command in a single interaction the dropping of a party; this implies that, at the SSF level, the object party is removed by the session, together with all its bearers and legs. To this aim, the SSF generates the appropriate messages towards all the BCSMs involved.

\subsection{The service control domain}

The service control domain realises the global handling of the service. The SCF, interacting with all the SSFs involved in the service, derives the overall network configuration by merging all the partial views represented by the single sessions.

The resultant model, called Global Logic Service Configuration is a high level one and it is designed to support the Service Logic Instance (SLI). It is worth noting that this model assumes fundamental relevance in case of a service realised adopting the distributed approach described above.

The GLSC is composed by four types of objects (Figure 4): i) the GLSC object, representing the active service instance; ii) the session object, identifying the session instance in each SSF involved in the service provisioning; iii) the actor object, representing either users or special resources (intelligent peripheral, bridge audio/video etc.) involved in the service; iv) the stream object, representing the information flow among actors. 
The actor and the stream objects hold the main parameters needed by the SLI. The actor object contains user specific characteristics, like the E.164 address, and their role in the service (e.g. optional or mandatory). The stream attributes concern the frame structure of the flow (data format and coding, e.g. MPEG1, JPEG), the quality of service and throughput values. The stream direction could be indicated by an attribute assigned to the relationship "is connected to", said attribute assuming the values incoming, outgoing or both.

The GLSC object model allows the SLI to retrieve all the necessary data. Thank to this model, it is possible to identify the session controlling the stream that connects two or more actors; therefore, if an actor has to be excluded from the service, it is straightforward to discriminate the session that the actor has joined and, within the session, the streams to which the actor is connected to.

It is to be noted that the more sophisticated is the service network configuration, the more important is the role played by the GLSC. This happens, for instance, when a great number of special resources are involved or when the service provisioning is carried out by means of the interaction with several SSPs.

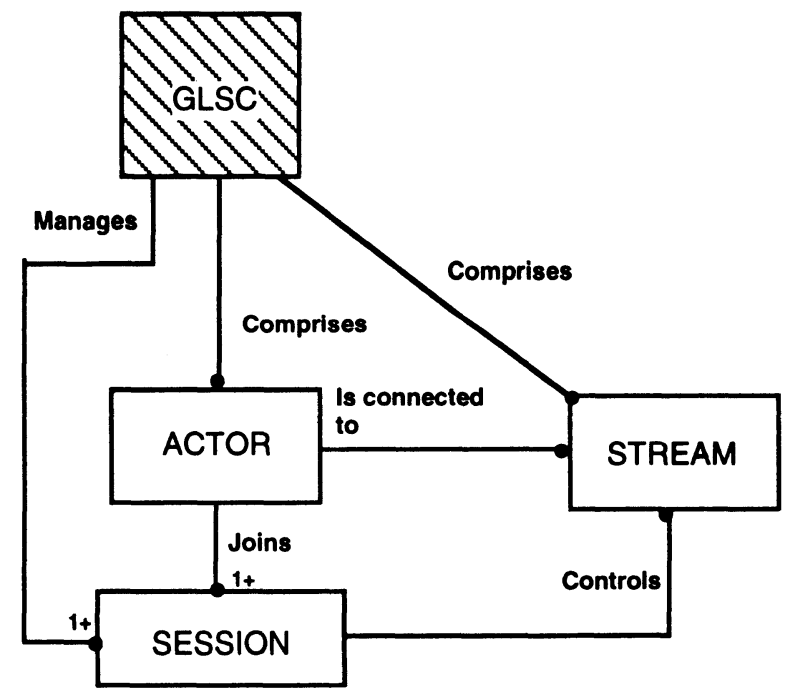

Figure 4 The GLSC object model.

\subsection{Interactions between session and service domains}

The typical interaction between the session domain and the service one (in the direction that goes from the former to the latter) is the following:

1. each event on the basic bearer connection is driven by the B-ISDN signalling and reported to the IN-SM by means of the Detection Points (DP) in the BCSM; 
2. the IN-SM modifies, in accordance to the event reported, the state of one or more objects represented in the session instance (for instance, the dynamic attribute representing the state of the bearer connection can be modified from being set-up to set-up);

3. the new state of the session is reported to the SCF and it is processed by the SLI;

4. according to the state being reported, the SLI updates the GLSC state.

In the opposite direction (from the service domain to the session one), an interaction is needed when, during the service processing, the SLI requires the session domain to execute an operation or when a user asks the SCF to execute a service procedure.

The steps of this interaction are:

1. the SLI retrieves the service network configuration held in the GLSC;

2. according to the information obtained and to the service procedure requested, the SLI commands one or more SSFs to execute the appropriate operation, by means of enhanced INAP messages mentioned above;

3. the IN-SMs add new objects or modify the state of those already present in their session instances, and the SSFs command the underlying CCFs to carry out the requested operations.

\section{THE BROADBAND-VIDEO CONFERENCE SERVICE}

In this section we apply the IN approach to support a multimedia-multiparty service, the Broadband Video Conference (B-VC).

The B-VC is a telecommunication service which allows end-to-end information transfers between two or more service subscribers [EURESCOM P506,1995]. This service provides the necessary arrangements for a real-time conferencing in which audio, video, and other media types can be exchanged among single individuals (single workstations) or group of individuals (located in conference rooms) via the B-ISDN.

The provision of this service requires very powerful signalling capability to obtain and manage a fully meshed audio/video interconnection among all the subscribers, making the B-VC one of the more complex services to be offered by the B-ISDN.

\subsection{Service description}

The B-VC service is provided by means of an "on demand" mode. The service consists of the following classes of procedures [AC068 INSIGNIA Deliverable]:

- procedures applicable to a not yet existing conference: conference creation;

- procedures applicable to an inactive conference: inactive conference modification, conference establishment; 
- procedures applicable to an active conference: addition of new conferee(s), disconnection of conferee(s), active conference modification, conference closing down.

The conference creation consists of a phase during which the user requiring the service, called hereafter conference coordinator, defines the conference profile: the media required, the list of users that could be involved and their profiles (e.g. role in the conference, procedures access rights, etc.). After the creation phase the conference can be activated immediately or later on.

During the inactive phase, the conference profile can be modified by the conference coordinator by means of the inactive conference modification procedure.

The conference establishment procedure allows the coordinator to start the active phase of the conference service; this procedure consist mainly in inviting the users and then in interconnecting them.

During the active phase of the conference a new conferee can be added to the service; this happens as a consequence of a request coming from an authorised conferee or directly from the new user. The coordinator can or can not accept this request. Of course, a conferee can leave an active conference anytime with the disconnection of conferee procedure (also, an authorised conferee can request the disconnection of another conferee).

The active conference modification procedure allows a user to request the coordinator to modify his/her profile; in addition the coordinator can use this procedure to indicate a possible new coordinator. Finally the conference closing can be obtained by means of the conference closing down procedure.

It is worth noting that the interaction between the users and the service logic can be supported by a Specialised Resource Function (SRF) located in a Broadband Intelligent Peripheral (B-IP); all the users are connected to this special resource via a data bearer and the B-IP interacts with the SLI by using the INAP protocol.

\subsection{Service analysis}

In this section we analyse the conference establishment procedure, realised both with the centralised architectural solution and with the distributed one, and compare the performance of the two approaches; we also study the resulting service representation models at the session domain and at the service domain.

The centralised approach is based on a relationship between a SCF and a single SSF. The latter is the SSF in which the conference coordinator has invoked the service logic.

The main steps to realise the service establishment are the following:

- the coordinator invokes, through a data bearer connected to the B-IP previously activated, the establishment procedure;

- the SCF sends to the SSP subsequent "SCP-initiated call" commands to realise a fully meshed interconnection of all the invited users;

- the SSF modifies the session view, orders the CCF to set-up the desired connections (bi-directional point-to-point or unidirectional point-to-multipoint) and reports the results to the SCF; 
- the SCF updates the GLSC according to the reported results.

Figure 5 represents the network configuration in the centralised approach for a three users conference.

The connections of the users to the B-IP are not shown; for the sake of neatness only one type of connection (for example the video one) is represented. As depicted in this figure, all the connections have to cross a fixed SSP, where are coordinated in a single session instance. The grey arrows represent the interactions between each independent BCSM and the IN-SM handling that BCSM. This solution can be applied for both the SCS 1 (Figure 5a) and the SCS 2.1 (Figure 5b).

This approach can lead to a very inefficient use of the network resources when the users involved are spread in a wide geographic area and thus can be very distant from the SSP of the conference coordinator.

To overcome this impairment the distributed approach can be used (Figure 6). The distributed approach leads to a multiple interaction between the SCF and two or more SSFs. The SSPs are chosen so as to obtain an efficient resource utilisation. The algorithm that optimises the resource utilisation is implemented in the SCP that realises the distribution of the connections among the sessions; the SCP commands the creation of new sessions, in addition to that already present in the SSP where the conference coordinator has required the execution of the service. As shown in Figure $6 \mathrm{a}$, the session 2 is created to handle the connections concerning users $\mathrm{B}$ and $\mathrm{C}$.

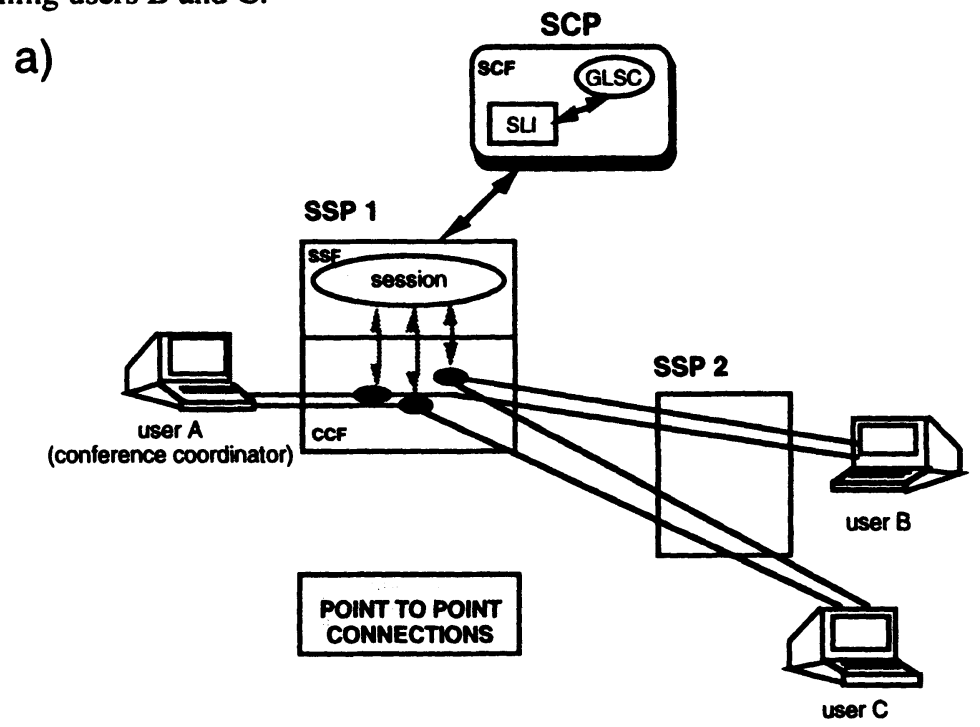

Figure 5a Network configuration in a centralised approach: case of point-to-point connections. 


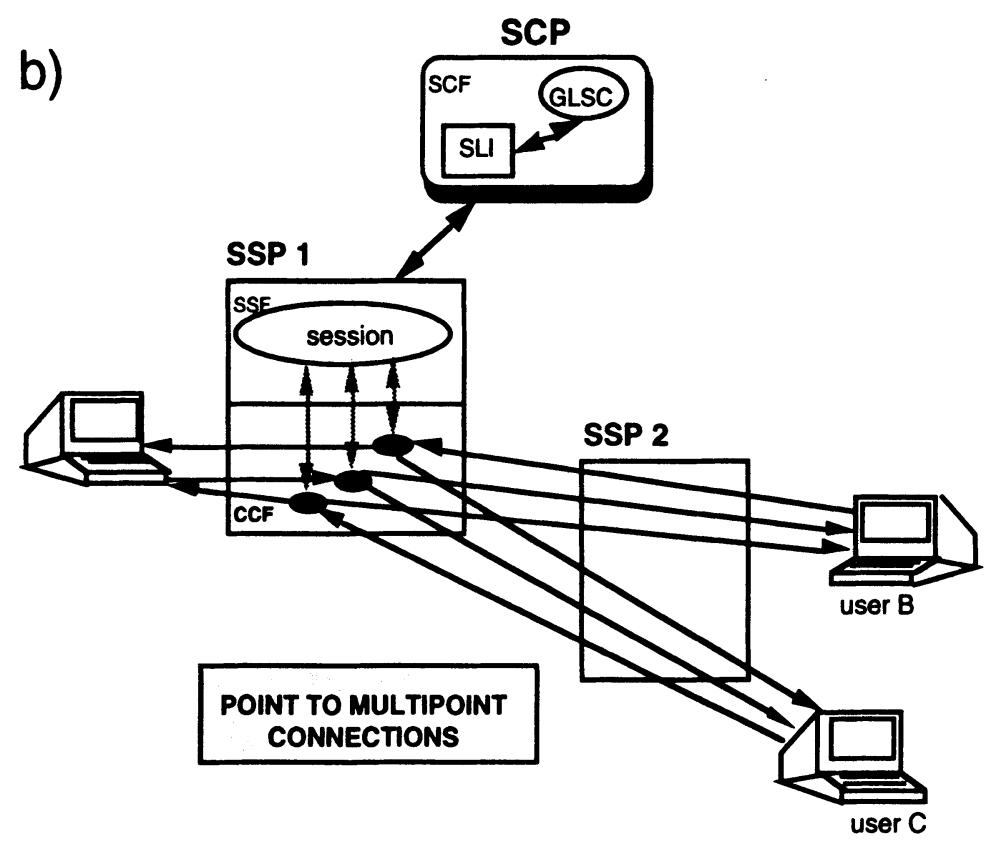

Figure 5b Network configuration in a centralised approach: case of point-to-multipoint connections.

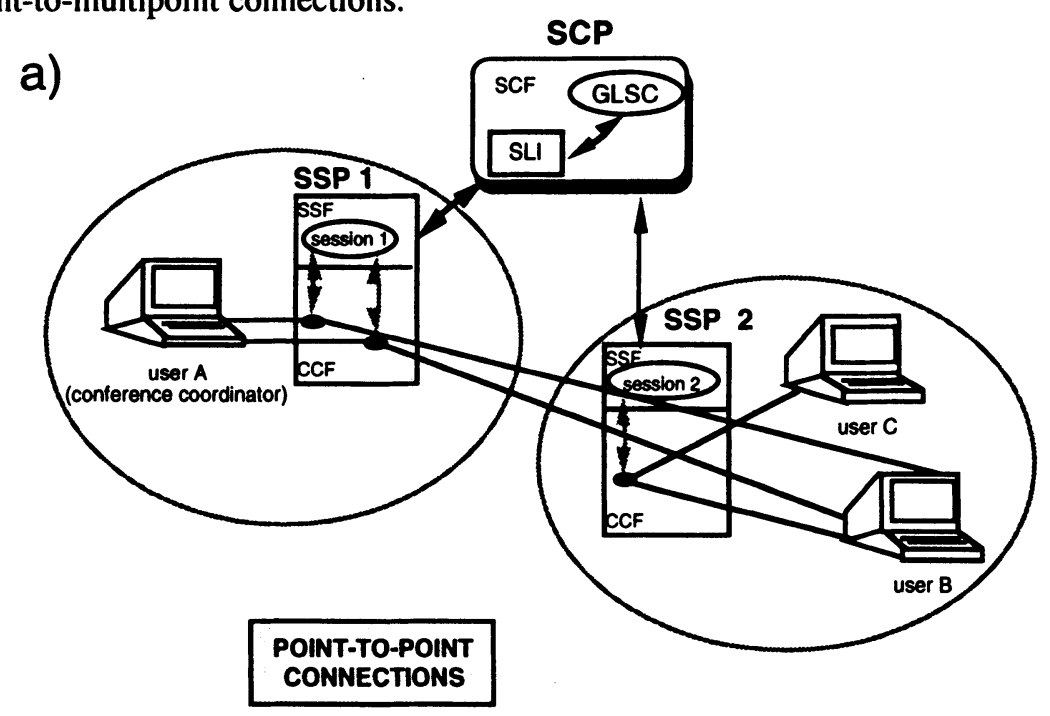

Figure 6a Network configuration in a distributed approach: case of point-to-point connections. 


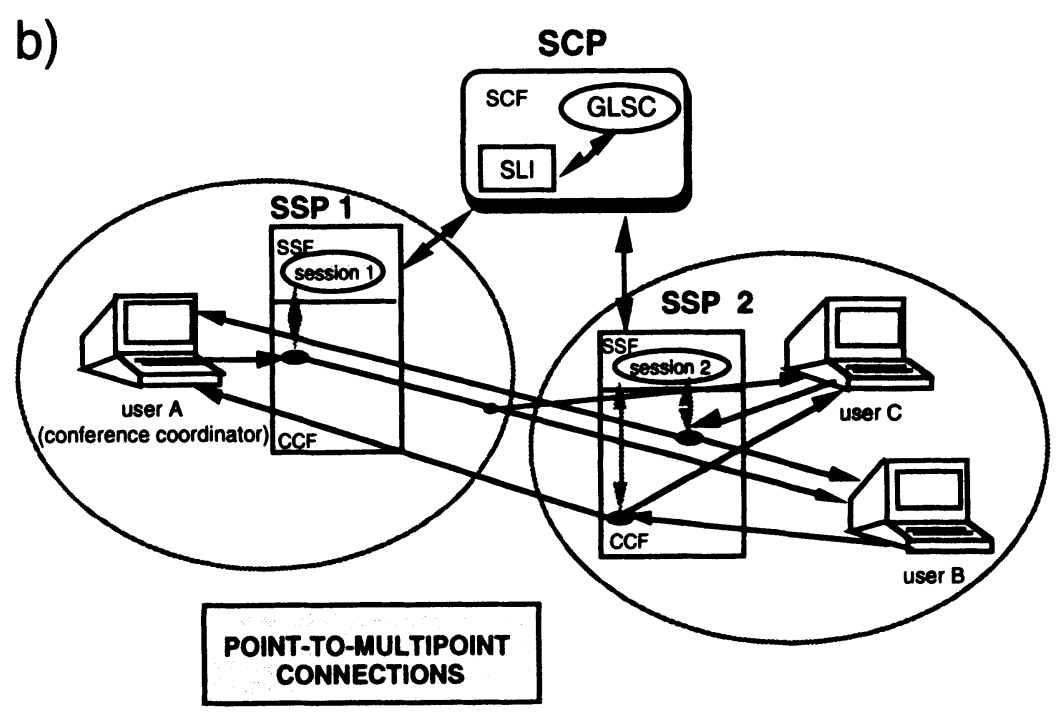

Figure 6b Network configuration in a distributed approach: case point-to-multipoint connections.

In order to compare the two approaches a performance study has been carried out. Two main aspects have been analysed: the signalling load on the network nodes and the transport resource utilisation.

Several network configurations with a variable number of SSPs and conferees have been considered. For a given network configuration we analysed all the possible associations between conferees and SSPs and the presence of a multiplicity of active conferences. Here only a snapshot of results is reported.

As far as the signalling load is concerned, we have analysed both the B-ISDN and the IN one. We have evaluated analytically the number of signalling messages handled by each network element during the realisation of the Video Conference control procedures. Such a number can be taken as a measure of the signalling load at the application level. With respect to the IN signalling load the performance study main results are the following:

- the application of the distributed approach does not increase in a significant way the total IN signalling load (just few messages more than the centralised approach, the ones required to create new sessions in the SSPs);

- the application of the distributed approach allows to reduce the processing load per SSP;

- the utilisation of point-to-multipoint connections implies a significance reduction of the IN messages exchanged (this result can be explained by considering that the number of the B-INAP messages is proportional to the number of connections that must be activated; that is $2 * \mathrm{~N}$ in the case of 
point-to-multipoint connections, $\mathrm{N}^{*}[\mathrm{~N}-1]$ in the case of point-to-point, if $\mathrm{N}$ is the number of conferees).

As regards the B-ISDN signalling load, in Figure 7 we report the number of messages as a function of the number of involved conferees. The comparison between distributed approach and centralised one shows that the former is cost effective both for point-to-point and for point-to-multipoint connections.

This vantage is due to the saving of signalling messages exchanged at the Network Node Interface.

Another result is that the adoption of point-to-point-connections determines a better performance behaviour with respect to the point-to-multipoint ones. This can be justified by considering that, even if the number of connections to be set-up in case of PMP is lower than the PP one $(2 * \mathrm{~N}$ vs. $\mathrm{N} *[\mathrm{~N}-1]$, where $\mathrm{N}$ is the number of conferees), the signalling messages exchanged for the set-up of a point-tomultipoint connection is proportional to the number of leafs and that one signalling relationship allows to handle a single versus of the communication.

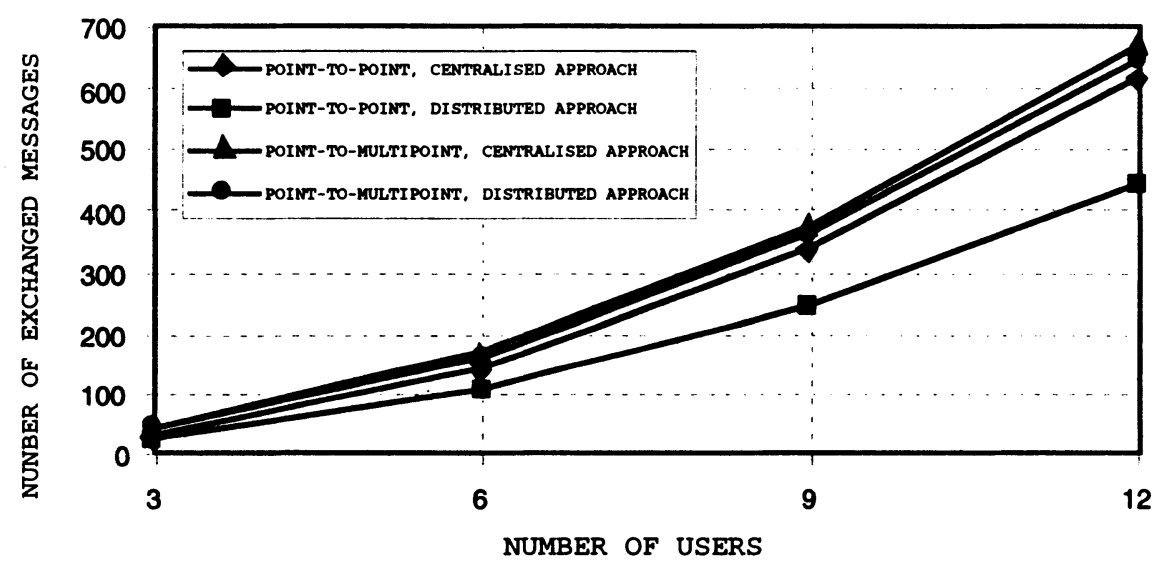

Figure 7 - Total mean B-ISDN load per SSP

To evaluate the transport resource utilisation we assumed a cost function that is linearly dependent on the mutual SSPs distance; in addition, direct connections between each couple of SSPs have been hypothesised.

Figure 8 shows the scheme of a case study network configuration. In this example six SSPs are located at the vertices of two triangles in such a way that the physical distance from a reference point in the centre of the configuration is R1 for the first three SSPs and R2 for the remaining ones. The actual links between the SSPs are not shown.

The rationale behind this choice is to describe a configuration corresponding to a first cluster of SSPs relatively close to each other, surrounded by additional SSPs located at a greater distance. By varying the above introduced parameters R1 and $\mathrm{R} 2$, a performance evaluation can be carried out, showing pros and cons of the 
centralised approach versus the distributed one, with respect to the transport resources utilisation.

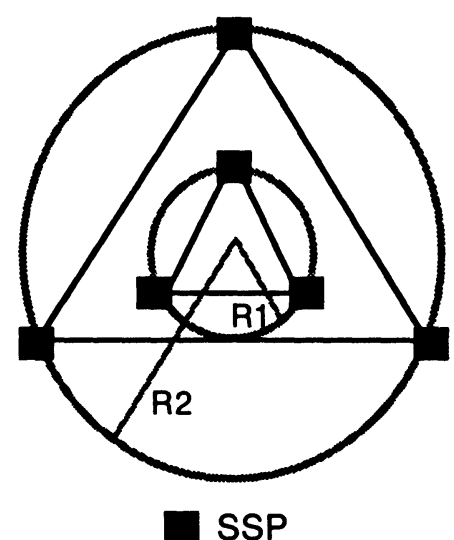

Figure 8 Scheme of the network configuration.

Figures 9 and 10 show the results arising from the analysis of the network configuration depicted in Figure 8, with $R 2 / R 1=100$. In Figure 9, the SSP relevant to conference coordinator is located on a vertex of the internal triangle, while, in Figure 10, it corresponds to a vertex of the external triangle.

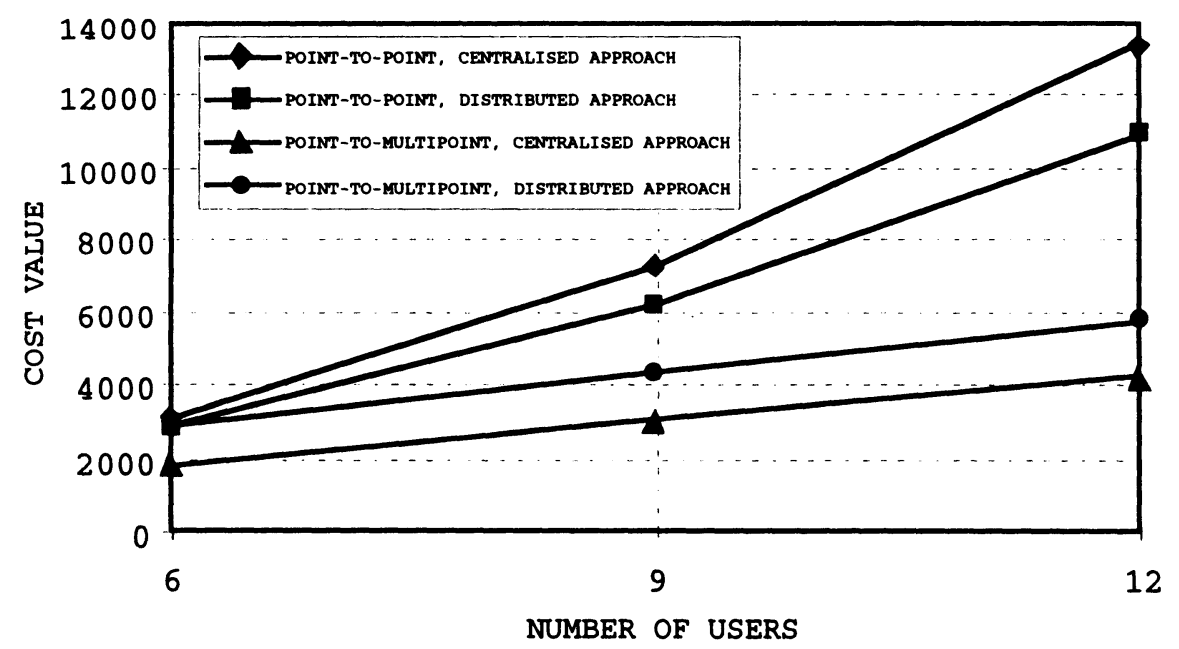

Figure 9 Cost value when the SSP relevant to the conference coordinator is located on the vertex of the internal triangle. 
As it can be noted, in the case of point-to-point connections the distributed approach is always very cost-effective with respect to the centralised approach, whereas if point-to-multipoint connections are used, the distributed approach is convenient only if the SSP relevant to the conference coordinator is far away from the "baricentric" position of the network configuration.

Finally, the greater is the number of users, the more convenient is the use of point-to-multipoint connections.

These results can be justified by considering that the distributed approach allows the interconnection of users belonging to the same SSP within the SSP itself. Moreover, in the case of point-to-multipoint connections, when a user relevant to a SSP has to be added to an active conference and a leg versus that SSP already exists, no further bandwidth has to be allocated. This is because in this case the cell replication function can be used.

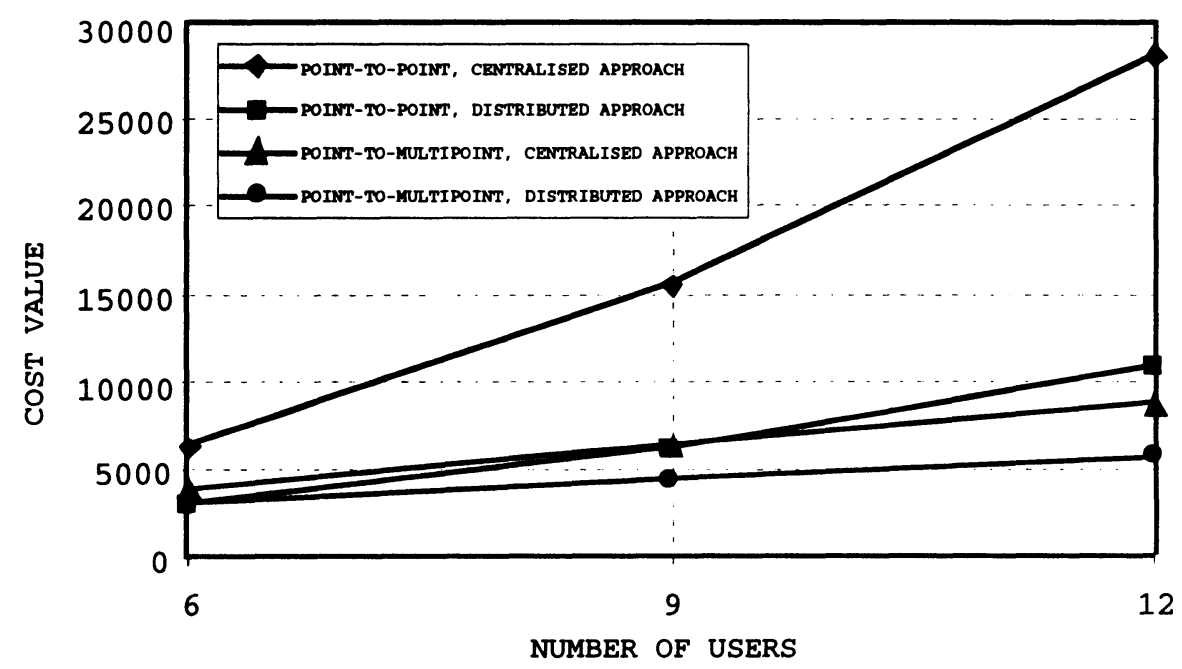

Figure 10 Cost value when the SSP relevant to the conference coordinator is located on the vertex of the external triangle.

\subsection{Example of the model instances in the B-VC}

In this section, we show an example of instances of the session model and of the service model. These examples refer to the service configuration of Figure 6a in which the distributed approach and point-to-point connections are assumed.

The upper part of Figure 11 shows the session instance in the SSP 1 after that the resulting connections are activated; the lower part represents the session 2 in the SSP 2. The session 1 instance models the connections between user A and users $B$ and $C$, whereas the session 2 instance models the local connection between users $B$ and $C$. 
It is to be noted that the owner of the session 2 is the SCP since this session is the result of an "SCP-activate session" command. In both the sessions the SCP is the owner of the connections activated by "SCP-initiated call" commands. The numbers labelling the objects are assigned in accordance to the temporal activation of the network components.

Figure 12 shows the GLSC instance. This instance represents the overall service configuration, comprising the three service users and the three video information flows. Moreover, the presence of the session object allows actors and streams to be associated to the relevant session. As it can be noted, each actor can belong to one or more sessions, while each stream belongs to one session only.
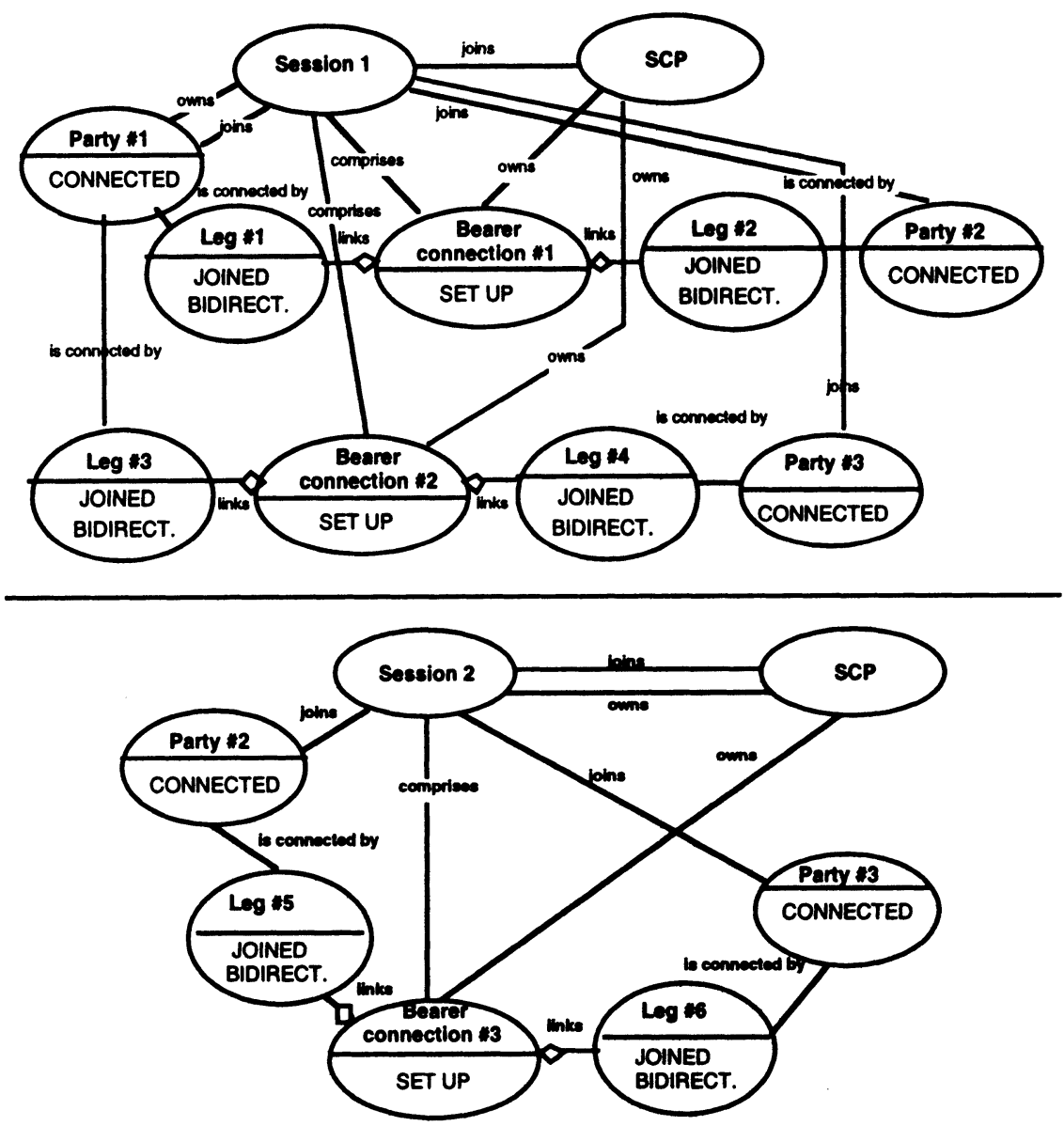

Figure 11 The session instances in SSP 1 and SSP 2. 


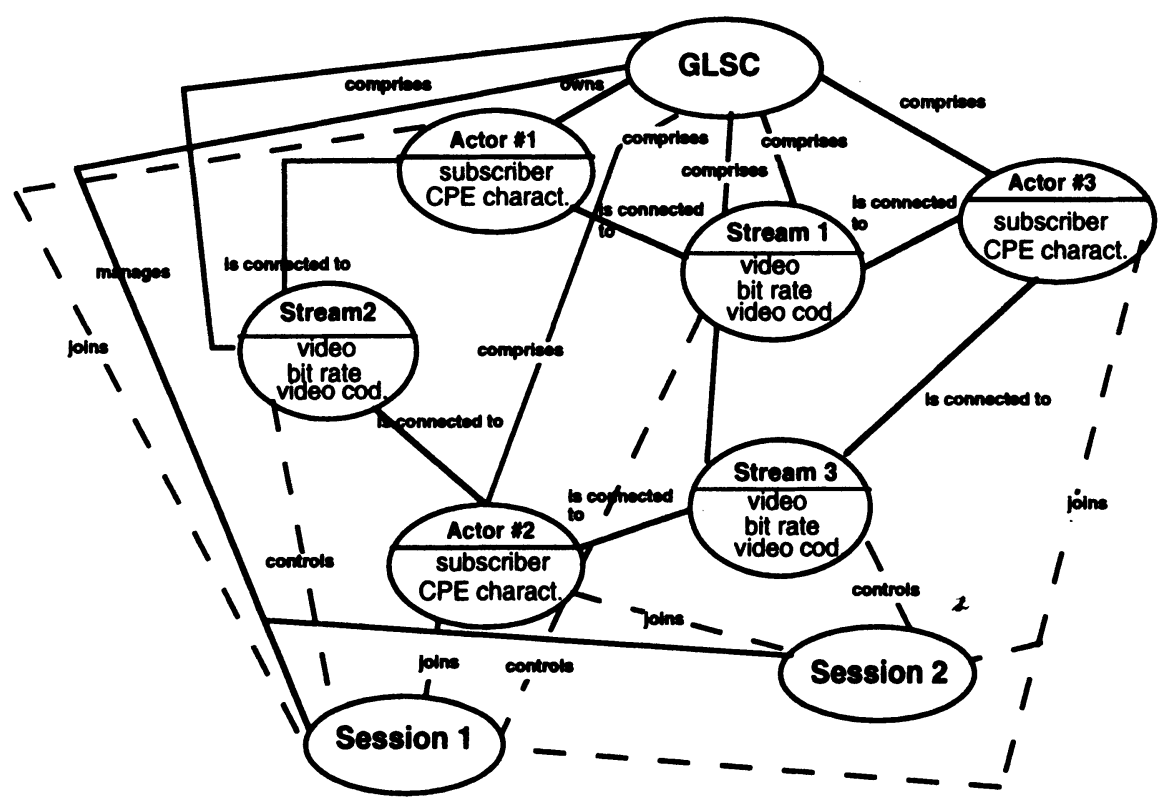

Figure 12 The GLSC instance in the SCP.

\section{CONCLUSIONS}

In this paper we proposed the introduction of a new functionality in the IN architecture, called Bearer Connections Coordinator and able to realise the provisioning of multimedia-multiparty services in cooperation with the B-ISDN. The solution here presented foresees that the Bearer Connections Coordinator functionality is shared between the Service Control Function and the Service Switching Function entities. Ad hoc object models have been defined for each functional level.

This approach has been applied for the provision of the Broadband Video Conference service, which requires very advanced control capability to handle the relevant network configuration. Finally, some performance results have been discussed in order to single out the main characteristics of the presented architectural solutions. 
AC068 INSIGNIA Deliverable (July 1995) Description of selected services.

AC068 INSIGNIA Deliverable (July 1996) First trial: Functions and Architecture Specification.

AC068 INSIGNIA Deliverable (July 1996) First trial: Protocol Specification

Carmagnola, V. Cuomo, F. and Ferretti, M. (June 1996 ) A layered approach for IN call modeling for the support of multimedia services in a B-ISDN environment, ICC '96, Vol. 2, 952-6.

Cuomo, F. Listanti, M. Ronchetti, L. and Salsano, S. (1996) Architectural alternatives for the support of a multipoint service in future B-ISDN, IIC International Institute of Communications, $43^{\circ}$ international conference of communication.

EURESCOM Project 506 HARMONISATION/INTEGRATION OF B-ISDN AND IN: PIR 1.1 (1995) Scenario for the introduction of Broadband Services and description of selected services.

van Maastricht, C and Schalk, E. (April 1995) Call modeling in a Broadband IN architecture, ISS'95, vol. 2, 340-4.

Mukasa, T. Ogino, N. Nakao, K. and Wakahara Y. (November 1995), Proposal for call modeling for Intelligent Network over Broadband ISDN, Globecom '95, 1265-71.

ITU-TS WP 1/11, SG 11, Q.2931 (June 1994) B-ISDN User Network Interface Layer 3 specification for Basic Call/Connection Control.

ITU-TS WP 1/11, SG 11, Q.2971 (September 1994), B-ISDN User Network Interface Layer 3 specification for Point-to multipoint Call/Connection Control.

ITU-TS Recommendations Q.1211-Q.1218 (1995), Intelligent Network CS-1.

Wakamoto, M. Fukazawa, M. Kim, M. W. and Murakami, K. (April 1995) Intelligent Network architecture with layered call model for multimedia-ondemand service, ISS '95, vol. 1, 201-5.

Francesca Cuomo received her Dr. Eng. degree in Electronics Engineering from the University "La Sapienza" of Roma in 1993. Since the beginning of 1993 she held a scholarship to work with the Telecommunication Networks Group at the INFOCOM Dept. of the University of Roma "La Sapienza". In November 1994 she entered a three years PhD program at the same Department. Since June 1996 she joined the INFOCOM Dept. as a Researcher in Communications, where she currently works in the area of Communications Networks.

Her current research interests focus on modelling and control of broadband networks and support of multimedia communications by means of the IN paradigm. 
Marco Listanti received his Dr. Eng. degree in Electronics Engineering from the University "La Sapienza" of Roma in 1980. He joined the Fondazione Ugo Bordoni in 1981, where has been leader of the TLC network architecture group until 1991. In November 1991 he joined the University of Roma, where he is currently an Associate Professor in Switching Systems. He also holds lectures at the University of Roma "Tor Vergata" on Communications Networks. His current research interests focus on multimedia broadband communications, high throughput switching architectures and integration between the Intelligent Network and the B-ISDN.

Fabrizio Pozzi graduated in Telecommunication Engineering at the University of Rome "La Sapienza" in 1996.

In the same year, he got a scholarship offered by Telecom Italia S.p.A. and his graduation thesis was carried out in the context of a collaboration program between the INFOCOM Dept. of the University of Rome "La Sapienza" and Telecom Italia S.p.A.

His research activities concerns the realisation of advanced telecommunication services by means of a B-ISDN/Intelligent Network integration.

He has Joined Telecom Italia S.p.A. since June 1997 as a member of the technical staff in the Business Customers Division. 